\title{
Unified theory of adsorption, pore-filling and wetting
}

\author{
M. W. Cole ${ }^{1}$ and E. S. Hernández ${ }^{2}$ \\ ${ }^{1}$ Department of Physics, 104 Davey Laboratory, \\ Pennsylvania State University, University Park, PA 16802 and \\ ${ }^{2}$ Departamento de Física, Facultad de Ciencias Exactas y Naturales, \\ Universidad de Buenos Aires, 1428 Buenos Aires, \\ and Consejo Nacional de Investigaciones Científicas y Técnicas, Argentina
}

Gases adsorb readily on surfaces and inside porous materials when there exists a sufficiently strong attraction provided by these materials. In the extreme opposite situation, little or no adsorption occurs when the attraction is weak. This paper derives a criterion distinguishing which of these two scenarios occurs at zero temperature. The calculations needed to solve these problems employ a set of simple models, adapted to a wide range of geometries. These include cylindrical, spherical and slit pores, corners formed at the intersection of flat surfaces, interstitial regions within nanotube bundles and flat surfaces. In each case, the distinguishing criterion is based on a small number of interaction parameters.

PACS numbers: $61.30 \mathrm{Hn}, 68.43 .-\mathrm{h}, 64.90 .+\mathrm{b}$

\section{INTRODUCTION}

Simple models have played an important role in statistical physics, often leading to robust qualitative conclusions about fundamental physical phenomena. Such models have been widely exploited to describe adsorption phenomena. A famous example is the lattice-gas application of the nearest-neighbor Ising model. Although this model oversimplifies the interaction, it yields exact critical exponents in two-dimensions (2D), confirmed by experiments for adsorbed gases. ? ? Similar experimental confirmations of solvable models' predictions have been found for the roughening transition (and the related layering transitions), the 3-states Potts model (applied to commensurate phases) and the Kosterlitz-Thouless theory of superfluid films.? ? ? ?

Our group has been concerned especially with wetting transitions.? Such transitions 
were predicted many years ago, ${ }^{?}$ using simple physical arguments as well as quite reliable models, and have been observed experimentally for a wide variety of gas/surface combinations.? ? ? ? ? ? ? ? ? In predicting these transitions, a so-called simple model has proved quite helpful in identifying candidate systems for such transitions and predicting the wetting temperature $T_{W}$. ? This prediction arises in implicit form when the free energy cost of adsorbing a film is compared with the energy gain associated with the gas-surface interaction. The model has been found to be semiquantitatively accurate by comparing predictions with results from experiments and computer simulations.?

With a similar goal of characterizing adsorbed films in simple terms, this paper addresses the problem of quasi-one-dimensional and quasi-two-dimensional adsorption in various geometries such as wedges, slits, cylinders andinterstitial channels. For each geometry, we ask whether a specific type of film occurs at chemical potential below that of the bulk ground state. The specific geometries considered here include quasi-one-dimensional and monolayer films, but do not include multilayer films, thus excluding some possible wetting transitions. If the film's chemical potential lies above that of the bulk, no such film phase occurs below saturation. Since the calculations presented here are classical, the term ground state means literally the state of lowest potential energy $E$ per particle. Our focus on the ground state is a restriction that allows us to exploit its known properties. An alternative approach might use the triple point for such a comparison.?

This paper is organized as follows. In Sec. II we present the very general considerations that lead us to establish a universal power-law rule for the condensation threshold of a classical fluid onto a two-or-three dimensional substrate. Specific geometries and results for particular adatom-substrate combinations are discussed in Sec. III. The discussion and summary is the subject of Sec. IV.

\section{UNIFIED DESCRIPTION OF THE ENERGETIC BALANCE}

Our general goal is to establish an equilibrium condition between a 3D state of bulk matter and a hypothetical one-dimensional (1D) or 2D system confined by an external potential. We specify the latter as created by either a smooth and continuous $2 \mathrm{D}$ sheet or by a $3 \mathrm{D}$ (bulk solid) source. The equilibrium coexistence condition of the adsorbed phase is that its energy per particle equals that of the ground state of the bulk material $E_{b u l k}$. The 
binding energy (chemical potential) of the adsorbed phase has additive contributions from adhesive and cohesive interactions, leading to this condition:

$$
E_{\text {cohesion }}^{D}+E_{\text {adhesion }}^{d}=E_{\text {bulk }}
$$

where $D=1,2$ is the dimensionality of the adsorbate phase and $d=2,3$ is that of the substrate. If the left side of this equation exceeds the right side, the bulk phase is favored and the adsorbed phase is absent at saturation; otherwise the adsorbed phase appears at, or below, the chemical potential the bulk material. Note that Eq. (1) represents an equilibrium condition, essentially different from the criterion of self-binding or positive cohesion of the adsorbate, where the right hand side should be set equal to zero.

One can establish various common characteristics of a large class of systems of interest. Neglecting many-body interactions, as is customary, the cohesive energy is attributed to a Lennard-Jones (LJ) pairwise interaction of strength $\varepsilon_{g g}$ and hard-core diameter $\sigma_{g g}$, and its ground-state value can be obtained by minimizing the total LJ potential energy with respect to the lattice constant. This leads to a dependence on the LJ well depth of the form

$$
E_{\text {cohesion }}^{D}=\alpha_{D} \varepsilon_{g g}
$$

with a coefficient $\alpha_{D}$ that contains specific details of the system dimensionality $D$. For the ground state crystalline configuration in $1 \mathrm{D}$ and $2 \mathrm{D}$, the optimum cohesive energies are, respectively

$$
\begin{aligned}
& E_{\text {cohesion }}^{1 D}=-1.03 \varepsilon_{g g} \\
& E_{\text {cohesion }}^{2 D}=-3.382 \varepsilon_{g g}
\end{aligned}
$$

In a similar way, one may think that the particles comprising the adsorbate interact with those of the substrate through another LJ potential with corresponding parameters $\varepsilon$ and $\sigma$. In the present study, we seek to optimize the cohesive energy by varying the geometry. The binding energy to the substrate is computed by minimization with respect to a parameter characterizing the geometry and is always of the form

$$
\begin{aligned}
E_{\text {adhesion }}^{d} & \propto \varepsilon \theta \sigma^{2} \quad \text { for } \mathrm{d}=2 \\
& \propto \varepsilon n \sigma^{3} \quad \text { for } \mathrm{d}=3
\end{aligned}
$$


where $\theta$ and $n$ respectively denote the areal density of a planar sheet or the bulk density of a continuum solid. In particular, the relation between areal density of a $2 \mathrm{D}$ substrate and the LJ hard-core parameter for atoms in the adsorber, $\sigma_{s s}$, is

$$
\begin{aligned}
\theta & =\left(\frac{2^{2 / 3}}{3^{1 / 2}}\right) \frac{1}{\sigma_{s s}^{2}} \\
& =\frac{0.916}{\sigma_{s s}^{2}}
\end{aligned}
$$

whereas for bulk material under the continuum approximation, one has

$$
\begin{aligned}
n & =\frac{\theta}{2^{1 / 6} \sigma_{s s}} \\
& =\frac{0.816}{\sigma_{s s}^{3}}
\end{aligned}
$$

We then see that the adhesive energy due to a substrate of dimension $d$ can be expressed as

$$
E_{\text {adhesion }}^{d}=\beta_{d} \varepsilon\left(\frac{\sigma}{\sigma_{s s}}\right)^{d}
$$

Finally, the energy per particle in the vapor phase is proportional to the gas-gas interaction strength, i.e.,

$$
E_{\text {vapor }}=\lambda \varepsilon_{g g}
$$

In particular, for a 3D LJ lattice one has $E_{b u l k}=-6.7 \varepsilon_{g g}$.

Collecting Eqs. (??), (??) and (??) into (??), we reach the universal rule

$$
\frac{\varepsilon_{g g}}{\varepsilon}=\frac{\beta_{d}}{\lambda-\alpha_{D}}\left(\frac{\sigma}{\sigma_{s s}}\right)^{d}
$$

This relation provides a threshold criterion for condensation and divides the parameter space into wetting (below) and nonwetting (above) regions. In other words, if a given combination of strength and hard-core parameters lies above the curve (??), the adsorbate does not condense on the specific substrate. The geometry of the confinement-i.e., cylinder, flat sheet or semi-infinite solid, wedge, interstitial channel-enters the coefficient $\beta_{d}$ and can be worked out for specific examples as shown in the next Section.

\section{SPECIFIC EXAMPLES}

In this Section, we illustrate the detailed form of the universal power law (??) for a variety of adatom-substrate combinations. We focus on the cases of (a) 1D matter within cylindrical 
pores created by rolling up a flat sheet and by drilling a cavity in a bulk material; (b) 2D matter on a planar sheet and on a semi-infinite solid. For completeness, we also examine condensation of 1D adsorbates in wedges and interstitial channels, and of 2D matter inside slit pores, i.e. monolayer films sandwiched between substrate half-spaces. We first note that according to Eqs. (3), (4) and the bulk value $E_{g g}=-6.7 \varepsilon_{g g}$, the threshold values of the adhesive energy are

$$
\begin{aligned}
& E_{\text {adhesion }}^{1 d}=-5.67 \varepsilon_{g g} \\
& E_{\text {adhesion }}^{2 d}=-3.318 \varepsilon_{g g}
\end{aligned}
$$

\section{A. One-dimensional matter $(D=1)$}

1. Two-dimensional substrates $(d=2)$.

The first example we consider is a hypothetical 1D phase (axial phase) of matter within a cylindrical tube made of a monolayer sheet of matter, e.g., a carbon nanotube. The potential energy on the cylinder axis is?

$$
V(0)=3 \pi^{2} \varepsilon \theta \sigma^{2}\left[\frac{21}{32}\left(\frac{\sigma}{R}\right)^{10}-\left(\frac{\sigma}{R}\right)^{4}\right]
$$

The optimal geometry (most strongly bound adsorbate) can be obtained by minimization with respect to the cylinder radius $R$ and corresponds to

$$
R_{\text {opt }}=\left(\frac{105}{64}\right)^{1 / 6} \sigma \approx 1.086 \sigma
$$

Consequently, the optimal potential energy is

$$
\begin{aligned}
E_{\text {adhesion }}^{2 d} & =-\frac{144 \pi^{2}}{5(105)^{2 / 3}} \varepsilon \theta \sigma^{2} \\
& =-12.8 \varepsilon \theta \sigma^{2} \\
& =-11.72 \varepsilon\left(\frac{\sigma}{\sigma_{s s}}\right)^{2}
\end{aligned}
$$

Condition (??) gives then

$$
\frac{\varepsilon_{g g}}{\varepsilon}=2.07\left(\frac{\sigma}{\sigma_{s s}}\right)^{2}
$$

We comment further about two other possible 1D phases originating from sheet-like substrates. One involves a wedge formed by two converging sheets. If the opening 
angle is small, there can be a strongly bound 1D queue of atoms parallel to the line of contact of the sheets. The adhesive energy in this small angle case is twice the value derived below for the case of a single planar sheet. The multiplicative factor becomes smaller than two as the opening angle of the wedge increases, but we do not evaluate this dependence here. The other related 1D phase is found at the interstitial channel in a nanotube bundle. The optimal energy in this case is three times the minimum energy provided outside of a single tube, since the coordination number is three. The actual value of this optimum energy depends on the radius of the tubes; for any specified radius the optimal spacing between tubes (which in general does not correspond to the actual spacing) can be determined.

2. Three-dimensional substrates $(d=3)$.

In this geometry one pore of interest is a cylindrical cavity. In this case, the potential energy on the cylinder axis is

$$
V(0)=\pi \varepsilon n \sigma^{3}\left[\frac{7}{32}\left(\frac{\sigma}{R}\right)^{9}-\left(\frac{\sigma}{R}\right)^{3}\right]
$$

and the optimal radius and potential energy are, respectively

$$
\begin{aligned}
R_{\text {opt }}=\left(\frac{21}{32}\right)^{1 / 6} \sigma \approx 0.932 \sigma \\
E_{\text {adhesion }}^{3 d}=-\frac{2}{3} \sqrt{\frac{32}{21}} \varepsilon n \sigma^{3} \\
=-2.59 \varepsilon n \sigma^{3} \\
=-2.11 \varepsilon\left(\frac{\sigma}{\sigma_{s s}}\right)^{3}
\end{aligned}
$$

Thus, from condition (??) we get

$$
\frac{\varepsilon_{g g}}{\varepsilon}=0.37\left(\frac{\sigma}{\sigma_{s s}}\right)^{3}
$$

For the wedge and interstitial channels, the same considerations hold as for the twodimensional substrates.

\section{B. Two-dimensional matter $(D=2)$}

1. Two-dimensional substrates $(d=2)$. 
Here the characteristic adsorbing substrate is a planar sheet, where the adsorbate experiences a potential energy of the form

$$
V(z)=2 \pi \varepsilon \theta \sigma^{2}\left[\frac{2}{5}\left(\frac{\sigma}{z}\right)^{10}-\left(\frac{\sigma}{z}\right)^{4}\right]
$$

with optimal distance and energy

$$
\begin{aligned}
& z_{\text {opt }}=\sigma \\
E_{\text {adhesion }}^{2 d} & =-\frac{6 \pi}{5} \varepsilon \theta \sigma^{2} \\
& =-3.77 \varepsilon \theta \sigma^{2} \\
& =-3.45 \varepsilon\left(\frac{\sigma}{\sigma_{s s}}\right)^{2}
\end{aligned}
$$

Condition (??) then gives

$$
\frac{\varepsilon_{g g}}{\varepsilon}=1.04\left(\frac{\sigma}{\sigma_{s s}}\right)^{2}
$$

2. Three-dimensional substrates $(d=3)$.

The confinement is now given by a semi-infinite solid with potential energy

$$
V(z)=\frac{2}{3} \pi \varepsilon n \sigma^{3}\left[\frac{2}{15}\left(\frac{\sigma}{z}\right)^{9}-\left(\frac{\sigma}{z}\right)^{3}\right]
$$

with optimal distance:

$$
z_{\text {opt }}=\sqrt{\frac{2}{5}} \sigma
$$

and optimal potential energy:

$$
\begin{aligned}
E_{\text {adhesion }}^{3 d} & =-\frac{2 \sqrt{10} \pi}{9} \varepsilon n \sigma^{3} \\
& =-2.21 \varepsilon n \sigma^{3} \\
& =-1.8 \varepsilon\left(\frac{\sigma}{\sigma_{s s}}\right)^{3}
\end{aligned}
$$

Condition (??) now gives

$$
\frac{\varepsilon_{g g}}{\varepsilon}=0.54\left(\frac{\sigma}{\sigma_{s s}}\right)^{3}
$$

It is worth noting that for matter in a planar slit made out of a $2 \mathrm{~d}$ or a $3 \mathrm{~d}$ material, the optimized potential energy is just twice that for a single wall. 


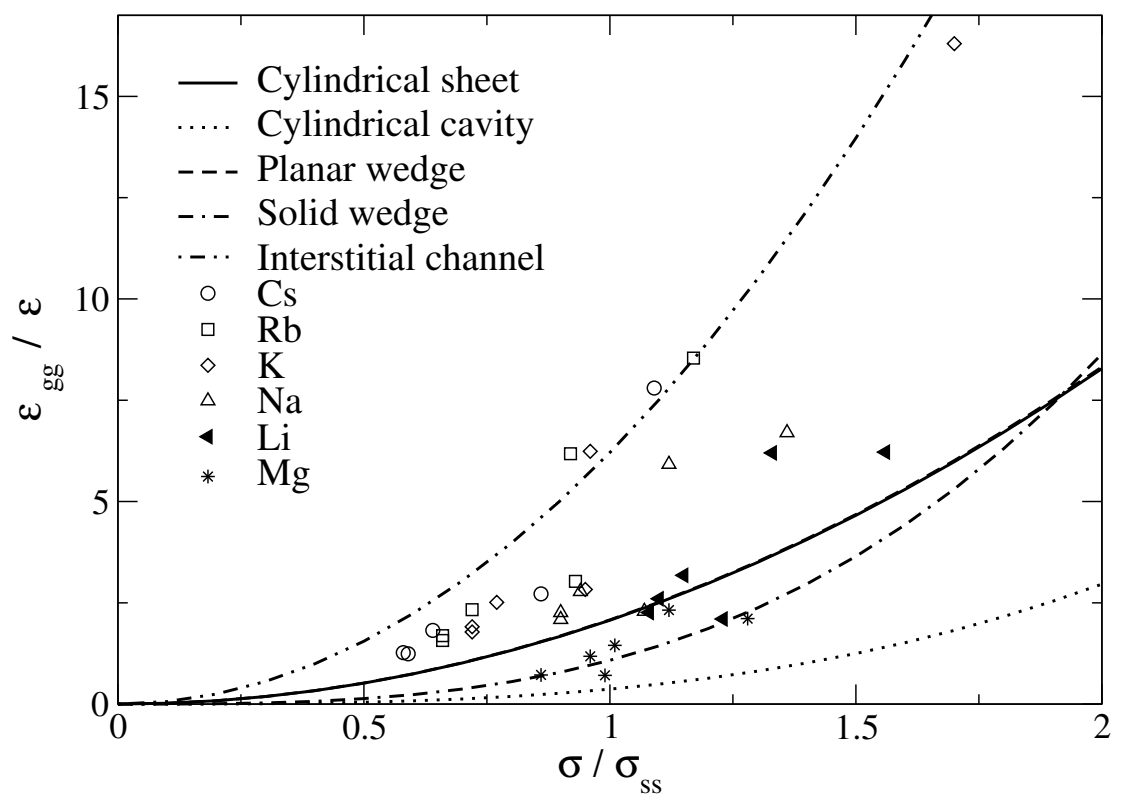

FIG. 1: The universal power-law for 1D matter in several environments. The points represent the adatom-substrate combinations whose parameters ratios are listed in Table 1.

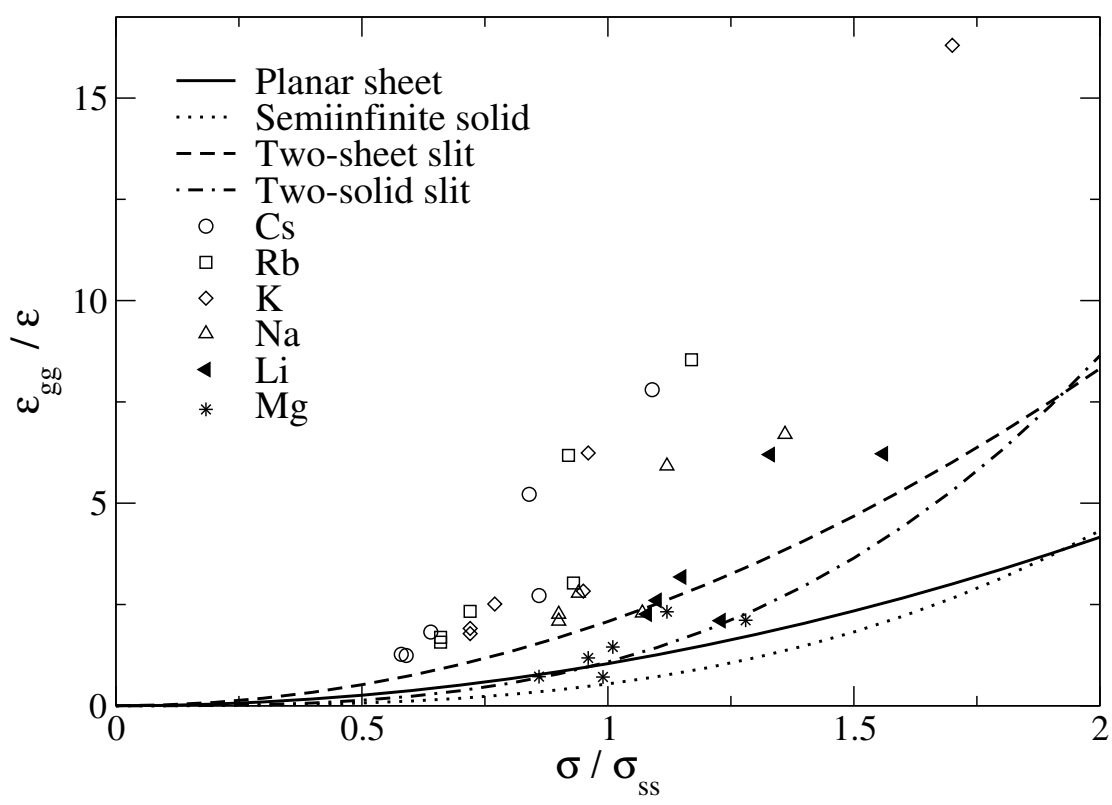

FIG. 2: Same as Fig. 1 for 2D matter. 
TABLE I: Ratios of LJ strengths and hard-core radii entering the universal law (??) for combinations of noble gases and hydrogen on alkali and alkaline earth metal substrates. The interactions are deduced from the gas-surface interactions of Ref. ? .

\begin{tabular}{|l|l|l|l|l|l|l|l|}
\hline & & $\mathrm{He}$ & $\mathrm{Ne}$ & $\mathrm{H}_{2}$ & $\mathrm{Ar}$ & $\mathrm{Kr}$ & $\mathrm{Xe}$ \\
\hline $\mathrm{Mg}$ & $\varepsilon_{g g} / \varepsilon$ & 2.11 & 2.32 & 0.71 & 1.45 & 1.18 & 0.72 \\
& $\sigma / \sigma_{s s}$ & 1.28 & 1.12 & 0.99 & 1.01 & 0.96 & 0.86 \\
\hline $\mathrm{Li}$ & $\varepsilon_{g g} / \varepsilon$ & 6.22 & 6.2 & 2.1 & 3.18 & 2.60 & 2.26 \\
& $\sigma / \sigma_{s s}$ & 1.56 & 1.33 & 1.23 & 1.15 & 1.10 & 1.08 \\
\hline $\mathrm{Na}$ & $\varepsilon_{g g} / \varepsilon$ & 6.7 & 5.92 & 2.29 & 2.78 & 2.26 & 2.09 \\
& $\sigma / \sigma_{s s}$ & 1.36 & 1.12 & 1.07 & 0.94 & 0.90 & 0.90 \\
\hline $\mathrm{K}$ & $\varepsilon_{g g} / \varepsilon$ & 16.3 & 6.24 & 2.83 & 2.51 & 1.91 & 1.78 \\
& $\sigma / \sigma_{s s}$ & 1.70 & 0.96 & 0.95 & 0.77 & 0.72 & 0.72 \\
\hline $\mathrm{Rb}$ & $\varepsilon_{g g} / \varepsilon$ & 8.54 & 6.18 & 3.03 & 2.33 & 1.69 & 1.57 \\
& $\sigma / \sigma_{s s}$ & 1.17 & 0.92 & 0.93 & 0.72 & 0.66 & 0.66 \\
\hline $\mathrm{Cs}$ & $\varepsilon_{g g} / \varepsilon$ & 7.80 & 5.22 & 2.72 & 1.82 & 1.27 & 1.24 \\
& $\sigma / \sigma_{s s}$ & 1.09 & 0.84 & 0.86 & 0.64 & 0.58 & 0.59 \\
\hline
\end{tabular}

We now exhibit in Figs. 1 and 2 the universal behavior for $1 \mathrm{D}$ and 2D adsorbates in various environments, respectively. Figure 1 displays the universal rule for a rolled-up cylindrical sheet and for a cylindrical cavity drilled within a solid material. For comparison, we also show the change in slope corresponding to planar and solid wedges and for an interstitial channel between three cylinders. The various points correspond to the LJ parameters for combinations of noble gases and hydrogen as adatoms, with alkali and alkaline earth metal substrates. Different symbols characterize various substrates; the specific numerical values of the parameters characterizing the noble gases and $\mathrm{H}_{2}$ are given in Table 1. Figure 2 displays the universal behavior of $2 \mathrm{D}$ matter on a planar surface corresponding to either a planar sheet or a semiinfinite substrate. For comparison, we also show the curves for corresponding slits. The points are the same as in Fig. 1. 


\section{DISCUSSION AND SUMMARY}

This paper presents the results of simple model calculations of the ground state of various phases of matter in different geometries. The figures permit conclusions to be drawn about whether a specific pair of gas/surface interaction parameters yields wetting vs. nonwetting, behavior. The selected systems, with parameters presented in these figures and the table, are physisorption systems that either exhibit wetting transitions on flat surfaces or are

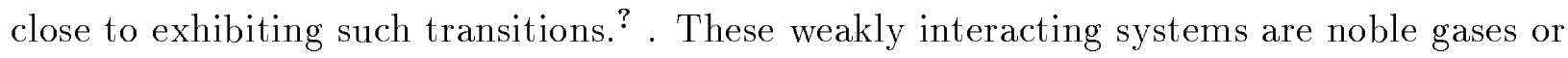

hydrogen near alkali or alkaline earth metal surfaces. There exist many other gas/surface combinations whose wetting behavior is not known, or less interesting because the adhesion is strong enough to ensure wetting in all geometries discussed here. (For example, the case of graphite would involve points close to the abscissa). Among the various geometries depicted in the two figures, the highest curve corresponds to the interstitial channel case. The reason is that the adhesive interaction is quite large, relatively speaking, and the cohesive interaction of the 1D phase is particularly small. Hence, all but a few of the systems shown exhibit wetting at $\mathrm{T}=0$. Among the other $1 \mathrm{D}$ phase geometries, the extreme opposite is the cylindrical cavity, which exhibits nonwetting for all the cases shown. One might wonder why a cylindrical cavity is less adhesive (more nonwetting) than a cylindrical sheet substrate. This difference is a consequence of our continuum substrate assumption. In a more realistic (discrete lattice) treatment of a multiwall nanotube, for example, the adhesive interaction would be greater than that of the single sheet and the opposite comparison would be drawn than one infers from the figure. Evidently, if one knows the actual interaction for such a specific case, one can make precisely the same kind of energetic comparison as that presented here in order to derive this more realistic conclusion. In comparing Figs 1 and 2, one observes many more points above the curves of figure 2. This means that many more $2 \mathrm{D}$ systems are nonwetting; the reason is the much larger (factor of 3) cohesive energy of the 2D phase. In such cases, wetting transitions are expected to occur at some finite temperature, except in a handful of ultraweakly interacting cases.? Computing this temperature is beyond the scope of the present paper. However, the simple model, described in the introduction, provides a means of estimating such a transition temperature; based on previous experience, ${ }^{?}$ we expect this prediction to have an accuracy of perhaps 20 As mentioned above, the present calculations derive a unified picture of wetting behavior in various geometries. The goal of 
universality was achieved by simplifying the interactions (as is commonly done) and focusing on temperature $\mathrm{T}=0$. Treating the more realistic case of finite $\mathrm{T}$ requires a significant increase in complexity of the calculations, but the qualitative behavior can be guided by the present results.

In comparing the present results with those of other calculations or experiments, it is useful to know the accuracy of the calculated energies, which is similar to that of other studies. As an example of $1 \mathrm{D}$ matter, we address the adsorption of $\mathrm{H}_{2}$ in carbon nanotubes, a much-studied system. Theoretical values of the binding energy per molecule are sensitive to the assumptions concerning the geometry and adsorption interactions, and, indeed, a wide variety of assumptions have been explored. A few general remarks about the effect of geometry can be made. One is that there is a possibility of considerable enhancement of the adsorption potential due to the high coordination within either a single nanotube or an interstitial channel within a nanotube bundle. For a cylindrical tube of optimized radius $R \approx$ $1.086 \sigma$, for example, the well depth of the adsorption potential is a factor $3 \pi / 2$ greater than the well depth on a planar sheet of the same material.? For a specific comparison with such an adhesive energy, the cohesive energy of the quantum ground state of $\mathrm{H}_{2}$ in mathematical $1 \mathrm{D}$ is just $4.8 \mathrm{~K} ;^{?}$ this value is a small fraction of the energy scale associated with the adsorption potential in strongly attractive environments. Inside an interstitial channel, for example, calculations find that the binding energy is increased to $500-600 \mathrm{~K}$, depending on whether or not dilation of the nanotube bundle is taken into account; such dilation, alone, increases the binding by nearly a factor of two.? The computed binding energy is particularly sensitive to the gas-surface interaction parameters in the case of interstitial adsorption. For example, a $2.5 \%$ decrease in the hard-core distance parameter increases the computed binding energy by $25 \%$, to more than $600 \mathrm{~K}$. These computed energy values are (linearly) sensitive to any uncertainty in the gas-substrate energy prefactor, which is usually derived from combining rules; these are known to have limited accuracy for gas phase interactions (see Ref. ? ). In addition, most calculations, like ours, employ an implicit assumption of additivity of gas-surface interactions, which neglects screening and solid state effects on the electrons within the nanotubes, as well as the effects of surface stress. ?

Finally, our calculations make the conventional approximation that the molecule is spherically symmetric. In contrast, some calculations and experimental data find large effects of 


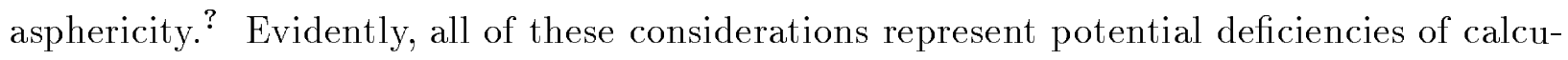
lated interactions near nanotubes.

These estimates of binding in various geometries may be compared with experimental results in some cases. For example, Wilson et al derived an experimental value of the isosteric heat $Q$ of nearly $900 \mathrm{~K}$ at the lowest coverage and a value near $400 \mathrm{~K}$ over a range of higher coverages. The latter value is consistent with predictions based on comparisons with adsorption on graphite, for which $Q$ and the binding energy are $499 \mathrm{~K}$ and $482 \mathrm{~K}$, respectively. However, no calculations of endohedral, groove or interstitial adsorption has yielded values of $Q$ comparable to the highest measured value, suggesting that the present model potentials are not quantitatively accurate.

\section{Acknowledgements}

This work was supported by grants DMR-0505160 from National Science Foundation, PID 5138/05 from Consejo Nacional de Investigaciones Científicas y Técnicas of Argentina and X298 from University of Buenos Aires. One of us (E. S. H.) is grateful for the American Physical Society for a Travel Award.

L. Onsager, Phys. Rev. 65, 117 (1944); C. N. Yang, Phys. Rev. 85, 808 (1952).

H. K. Kim and M. H. W. Chan, Phys. Rev. Lett. 53, 170 (1984).

L. W. Bruch, M. W. Cole and E. Zaremba, Physical Adsorption: Forces and Phenomena (Oxford, 1997).

B. Nienhuis, A. N. Berker, E. Riedel and M. Schick, Phys. Rev. Lett. 43, 737 (1979).

J. G. Dash, M. Schick and O. E. Vilches, Surf. Sci. 299/300, 405 (1994).

J. M. Kosterlitz and D. J. Thouless, Prog. Low Temp. Phys. Vol. 7B (Plenum, NY, 1973), pp. 193-231.

E. Cheng, M. W. Cole, J. Dupont-Roc, W. F. Saam and J. Treiner, Rev. Mod. Phys. 65, 557 (1993).

J. W. Cahn, J. Chem. Phys. 66, 3667 (1977). 
C. Ebner and W. F. Saam, Phys. Rev. Lett. 38, 1486 (1977).

D. Bonn and D. Ross, Rep. Prog. Phys. 64, 1085 (2001).

R. Pandit, M. Schick and M. Wortis, Phys. Rev. B 26, 5112 (1982).

D. E. Sullivan and M. M. Telo da Gama, in Fluid Interfacial Phenomena, ed. C. A. Croxton (Wiley, NY, 1985).

S. Dietrich, in Phase Transitions and Critical Phenomena, Vol.12, ed. C. Domb and J. Lebowitz (Academic Press, London, 1987).

P.-J. Nacher and J. Dupont-Roc, Phys. Rev. Lett. 67, 2966 (1991).

J. Rutledge and P. Taborek, J. Low Temp. Phys. 95, 405 (1994).

K. S. Ketola, S. Wang and R. B. Hallock, Phys. Rev. Lett. 68, 201 (1992); R. B. Hallock, J. Low Temp. Phys. 101, 31 (1995).

G. Mistura, H. C. Lee and M. H. W. Chan, J. Low Temp. Phys. 96, 221 (1994).

G. B. Hess, M. J. Sabatini and M. H. W. Chan, Phys. Rev. Lett. 78, 1739 (1998).

F. Hensel and M. Yao, Eur. J. Sol. St. Inorganic Chem. 34, 861 (1997).

V. F. Kozhevnikov et al, Fluid Phase Equilibria 150-151, 625 (1998).

E. Cheng, M. W. Cole and W. F. Saam and J. Treiner, Phys. Rev. Lett. 67, 1007 (1991).

S. Curtarolo, G. Stan, M. J. Bojan, M. W. Cole, and W. A. Steele, Phys. Rev. E 61, 1670 $(2000)$.

G. Stan and M. W. Cole, Surf. Sci. 395, 280 (1998).

A. Chizmeshya, M. W. Cole, and E. Zaremba, J. Low Temp. Phys. 110, 677 (1998)

F. Ancilotto, S. Curtarolo, F.Toigo and M. W. Cole, Phys. Rev. Lett. 87, 206103 (2001)

M.C. Gordillo, J. Boronat, and J. Casulleras, Phys. Rev. B 61, R878 (2000).

M.M. Calbi, F. Toigo, and M.W. Cole, Phys. Rev. Lett. 86, 5062 (2001) and J. Low Temp. Phys. 126, 179 (2002).

M.C. Gordillo, J. Boronat, and J. Casulleras, Phys. Rev. B 68, 125421 (2003).

G. Scoles, Intl. J. Quantum Chem. 24, 475 (1990).

M. M. Calbi, A. Mizel and M. W. Cole, Phys. Rev. B 69, 195408 (2004).

M. K. Kostov, M. W. Cole, J. C. Lewis, P. Diep and J. K. Johnson, J. Chem. Phys. 116, 1720 (2002)]; M. K. Kostov, H. Cheng, R. M. Herman, M. W. Cole and J. C. Lewis, Chem. Phys. Lett. 332, 26-34 (2000).

T. Wilson, A. Tyburski, M. R. DePies, O. E. Vilches, D. Becquet and M. Bienfait, J. Low 
Temp. Phys. 126, 403 (2002).

G. Vidali, G. Ihm, H. Y. Kim and M. W. Cole, Surf. Sci. Reports. 12, 135 (1991). 